\title{
Whole brain inhomogeneous Magnetization Transfer (ihMT) imaging: sensitivity enhancement within a steady-state gradient echo sequence
}

S. Mchinda ${ }^{1}$, G. Varma ${ }^{2}$, V.H. Prevost ${ }^{1}$, A. Le Troter $^{1}$, S. Rapacchi ${ }^{1}$, M. Guye ${ }^{1,3}$, J. Pelletier ${ }^{1,4}$, J-P Ranjeva $^{1}$, D.C Alsop ${ }^{2}$, G. Duhamel ${ }^{1}$, O.M. Girard ${ }^{1}$

${ }^{1}$ Aix Marseille Univ, CNRS, CRMBM UMR 7339, Marseille, France

${ }^{2}$ Radiology, Division of MR Research, Beth Israel Deaconess Medical Center, Harvard Medical School, Boston, MA, United States

${ }^{3}$ Aix Marseille Univ, APHM, Hôpital de La Timone, Pôle d'Imagerie Médicale, CEMEREM, Marseille, France

${ }^{4}$ Aix Marseille Univ, APHM, Hôpital de La Timone, Pôle de Neurosciences Cliniques, Service de Neurologie, Marseille, France

Corresponding author:

Olivier M. Girard, PhD

Aix-Marseille Université

Centre de Résonance Magnétique Biologique et Médicale CRMBM - CEMEREM, UMR 7339 CNRS - Faculté de Médecine

27 Boulevard Jean Moulin, 13005 Marseille, France

E-mail : olivier.girard@univ-amu.fr

ORCID: 0000-0002-5296-638X

Twitter: @OlivierMGirard

Paper type: full paper for MRM

Word Count: 5998

Number of figures: 8

Number of supplementary figures: 7

Number of tables: 2

Running head:

Whole Brain Inhomogeneous Magnetization Transfer (ihMT) 


\section{ABSTRACT (max 200 words)}

Purpose: To implement, characterize and optimize an interleaved inhomogeneous magnetization transfer (ihMT) gradient echo (GRE) sequence allowing for whole-brain imaging within a clinically compatible scan time.

Theory and Methods: A general framework for ihMT modelling was developed based on the Provotorov theory of radiofrequency saturation, which accounts for the dipolar order underpinning the ihMT effect. Experimental studies and numerical simulations were performed to characterize and optimize the ihMTGRE dependency with sequence timings, saturation power and offset frequency. The protocol was optimized in terms of maximum signal intensity and the reproducibility assessed for a nominal resolution of $1.5 \mathrm{~mm}$ isotropic. All experiments were performed on healthy volunteers at $1.5 \mathrm{~T}$.

Results: An important mechanism driving signal optimization and leading to strong ihMT signal enhancement has been identified, that relies on the dynamics of RF energy deposition. By taking advantage of the delay allowed for readout between ihMT pulse bursts it was possible to boost the ihMT signal by almost 2 fold compared to previous implementation. Reproducibility of the optimal protocol was very good, with an intra-individual error $<2 \%$.

Conclusion: The proposed sensitivity-boosted and time-efficient steady-state ihMT-GRE sequence implemented and optimized at 1.5T, allowed robust high-resolution 3D ihMT imaging of the whole brain within a clinically compatible scan time.

Keywords: Inhomogeneous Magnetization Transfer, ihMT, Provotorov theory of radiofrequency saturation, Myelin, dual frequency RF saturation, dipolar order, Magnetization Transfer model. 


\section{INTRODUCTION}

Inhomogeneous magnetization transfer (ihMT) is a recent MRI technique derived from conventional magnetization transfer (MT) that has shown promise for myelin imaging (1-3). Conventional MT is sensitive to tissue macromolecular content by selective saturation of the macromolecular proton pool and subsequent saturation transfer to the detectable mobile proton signal. IhMT exploits the signal difference obtained when comparing two simple MT experiments performed with the same RF power: one using single-frequency radiofrequency (RF) saturation and another using dual-frequency RF saturation at two symmetric offset frequencies. Single frequency RF irradiation produces spectrally nonuniform and asymmetric RF saturation effects (4) characteristic of the underlying dipolar order of broad macromolecular lines. Conversely, the dual frequency RF irradiation effectively decouples Zeeman and dipolar orders and provides a symmetric and more efficient RF saturation scheme (5). Hence, by focusing on the difference between these two saturation schemes, ihMT reveals dipolar order. MT experiments have been traditionally achieved using single frequency RF and although the contribution of dipolar order has been acknowledged in a few studies (6-8), it is conventionally dismissed from quantitative MT (qMT) analysis $(9,10)$ because it is found to compromise qMT parameter precision. Following the discovery of the ihMT effect (1) the fundamental role of dipolar order in the ihMT signal has been fully appreciated and included in a theoretical model combining single- and dual-frequency RF irradiation experiments according to the Provotorov theory of RF saturation (11). IhMT should be envisioned as a measure of the dipolar order underlying broad macromolecular lines and as such, provides access to a new source of contrast based on the dipolar order relaxation time, $T_{1 D}$.

The molecular origin of the ihMT effect is complex. IhMT arises from specific molecular architecture and water access conditions to macromolecular protons that preserve dipolar order. Intense inMT signal requires strong residual dipolar coupling to give rise to broad resonance lines, but also requires spin diffusion to be relatively slow as compared to the time-scale of RF saturation, in order to prevent fast relaxation of the dipolar order. IhMT is prominent in lipid systems arranged in a liquid crystalline phase and is naturally influenced by molecular mobility and temperature conditions that modulate motional averaging mechanisms (12). Whereas ihMT signal typically increases with $T_{1 D}$, the formal relationship between dipolar broadening, the specific averaging mechanisms, the resulting lineshape and the strength of the ihMT signal is still an open question. Inhomogeneous broadening is not a prerequisite for non-uniform saturation to occur (13) from dipolar broadened lines. However the investigated homogeneously broadened systems exhibited shorter $\mathrm{T}_{1 \mathrm{D}}$ as compared to inhomogeneously broadened 
ones (13) and it remains plausible that inhomogeneous broadening associated with fast rotating lipid chains $(14,15)$ within biological membranes could explain the higher ihMT signal observed in myelinated tissues. In any case, the spectrally non-uniform RF saturation effect caused by the underlying dipolar order when using single frequency RF is the key mechanism allowing sensitization of inMT to dipolar order. The name inhomogeneous magnetization transfer was intended to reflect this characteristic feature, which was not fully appreciated under the conventional MT paradigm.

Previous ihMT implementations have mostly consisted of long trains of soft RF saturation pulses followed by fast readout modules (1-3,16-19). In the following study we refer to these kinds of implementation as ihMT-prep sequences. Within this framework, a long recovery time typically follows readout to restore longitudinal magnetization for the next acquisition, hence penalizing the timeefficiency of the sequence. IhMT-prep sequences are suitable for 2D imaging with a limited number of slices, but alternative strategies are required for whole brain ihMT imaging. Preliminary reports have demonstrated the feasibility of 3D whole brain ihMT within a clinically compatible scan time by applying short bursts of RF saturation pulses between gradient echo (GRE) readouts $(20,21)$. This elementary sequence kernel (RF burst + readout) is repeated until a steady state is reached and may be repeated as long as necessary to acquire a whole 3D volume, as for conventional MT (22). We refer to this kind of approach as ihMT-GRE in the following. Of interest, the ihMT-GRE sequence intrinsically carries an additional temporal degree of freedom as compared to the ihMT-prep variants. Indeed, the readout duration defines a mixing period during which relaxation and magnetization transfer occurs in between successive RF bursts. This mechanism has been exploited to enhance ihMT signal intensity in a recent preliminary study (23) and subsequently dubbed the boost-effect. The current study builds upon these recent findings with the following aims: $1 /$ to establish a framework to simulate ihMT-GRE sequences and model ihMT dependence on sequence parameters; 2/ to characterize ihMT dependence on the saturation parameters and to optimize the RF energy deposition scheme to achieve the boost-effect; and 3 / to acquire whole brain sensitivity-boosted ihMT-GRE images within a clinically compatible scan time at $1.5 T$. 


\section{THEORY}

Single- and dual-frequency MT effects can be modeled using spin temperature concepts to describe the time-evolution of the free and bound pool magnetizations. The inMT signal is calculated by subtracting the free pool magnetization following single and dual-frequency RF irradiation. IhMT modeling requires that the bound pool includes a dipolar reservoir in addition to the Zeeman reservoir (11). This dipolar reservoir is represented by a dipolar spin temperature and characterized by a dipolar relaxation time $T_{1 \mathrm{D}}$. Dipolar order tends to reduce RF saturation effects when single frequency irradiation is applied according to the Provotorov theory of RF saturation, Eq.1:

$\frac{d M_{Z A}}{d t}=R_{1 A}\left(M_{Z A}^{0}-M_{Z A}(t)\right)-R M_{Z B}^{0} M_{Z A}(t)+R M_{Z A}^{0} M_{Z B}(t)-R_{R F A} M_{Z A}(t)$

$\frac{d M_{Z B}}{d t}=R_{1 B}\left(M_{Z B}^{0}-M_{Z B}(t)\right)-R M_{Z A}^{0} M_{Z B}(t)+R M_{Z B}^{0} M_{Z A}(t)-R_{R F B} M_{Z B}(t)+R_{R F B} \beta^{\prime}(t)$

$\frac{d \beta^{\prime}}{d t}=-\frac{1}{T_{1 D}} \beta^{\prime}(t)+R_{R F B}\left(\frac{2 \pi \Delta f}{D}\right)^{2}\left(M_{Z B}(t)-\beta^{\prime}(t)\right)$

where $M_{Z A}$ and $M_{Z B}$ are the longitudinal magnetizations (i.e. Zeeman orders) of the free and bound pools, respectively; $M_{Z A}^{0}$ and $M_{Z B}^{0}$ are the corresponding equilibrium magnetization, $\mathrm{R}_{1 \mathrm{~A}}$ and $\mathrm{R}_{1 \mathrm{~B}}$ the corresponding longitudinal relaxation rates and $\mathrm{R}$ the exchange rate between the two pools. $\beta$ is the dipolar spin temperature, with $\beta^{\prime}=\Delta f \beta$, and $\Delta f$ the frequency offset of RF saturation. $R_{R F A}$ and $R_{R F B}$ correspond to the RF absorption terms of the free and bound pools, respectively, calculated as $R_{R F i}=\pi \omega_{1}^{2} g_{i}(2 \pi \Delta f), i=A$ or $B$, with $\omega_{1}=\gamma B_{1}$ the RF amplitude and $g_{i}$ the absorption lineshapes (assumed to be Lorentzian for the free pool, and super-Lorentzian for the bound pool $(6,9,11)$ ) and characterized by their corresponding transverse relaxation times $T_{2 A}$ and $T_{2 B}$. Finally $D$ is the local dipolar field; a parameter related to the second moment of the lineshape and calculated as $1 /\left(T_{2 B} \sqrt{15}\right)$ for a super-Lorentzian (6).

Under symmetric dual frequency irradiation, the coupling between Zeeman and dipolar orders effectively vanishes (11) so that Eq.1 reduces to the classical binary spin bath model with an additional independent equation describing relaxation and saturation effects of the dipolar order, Eq.2:

$$
\begin{aligned}
& \frac{d M_{Z A}}{d t}=R_{1 A}\left(M_{Z A}^{0}-M_{Z A}(t)\right)-R M_{Z B}^{0} M_{Z A}(t)+R M_{Z A}^{0} M_{Z B}(t)-R_{R F A} M_{Z A}(t) \\
& \frac{d M_{Z B}}{d t}=R_{1 B}\left(M_{Z B}^{0}-M_{Z B}(t)\right)-R M_{Z A}^{0} M_{Z B}(t)+R M_{Z B}^{0} M_{Z A}(t)-R_{R F B} M_{Z B}(t)
\end{aligned}
$$


$\frac{d \beta^{\prime}}{d t}=-\frac{1}{T_{1 D}} \beta^{\prime}(t)-R_{R F B}\left(\frac{2 \pi \Delta f}{D}\right)^{2} \beta^{\prime}(t)$

Finally, the time dependent ihMT signal may be calculated from the free-water magnetization as Eq.3:

$i h M T(t)=M_{Z A}^{+}(t)+M_{Z A}^{-}(t)-2 M_{Z A}^{+/-}(t)$ and $\operatorname{ihMTR}(t)=i h M T(t) / M_{Z A}^{R F=0}(t)$

Where $M_{Z A}^{+}$corresponds to a single RF saturation applied at $+\Delta f$ offset, $M_{Z A}^{+/-}$corresponds to a dual RF saturation applied at $\pm \Delta f$ offsets, and $M_{Z A}^{R F=0}$ corresponds to a RF saturation power set to zero (usual GRE signal). Eq.3 includes a complementary symmetrical saturation condition $\left(M_{Z A}^{-}\right.$corresponding to a single RF saturation applied at $-\Delta f$ offset) to provide first order correction of MT asymmetry effects induced by the slight chemical shift of the macromolecular line, which was shown to be effective even for preclinical applications at ultra-high field (17).

\section{METHODS}

All experiments were performed on a whole body 1.5T MRI system (Avanto, Siemens, Erlangen, Germany) with body coil transmission and a 12-channel receive-only head coil on healthy volunteers (22 total, including 8 women; mean age: $26.1 \pm 7.9$ years). Experiments were approved by the institutional ethics committee on clinical investigations (CRMBM, Marseilles), and written informed consent was obtained from each participant before the study.

\section{Pulsed ihMT sequence implementation}

Dual frequency RF saturation can be achieved using a cosine-modulation of MT saturation pulses or by fast switching the carrier frequency of consecutive MT pulses $(1,19)$. Here a pulsed ihMT module with frequency-alternating dual-saturation was interleaved with a GRE readout (Fig.1) $(21,23)$ leading to a sequence kernel of $N_{p}$ consecutive saturation pulses (Hann-shaped, unless otherwise specified) followed by data acquisition. For long repetition times (TRs) the total acquisition time of multiple 3D volumes may become prohibitively long and clinically unfeasible. Thus readout segmentation was implemented to speed-up scan time, allowing acquisition of several lines of k-space, or segments, in between MT pulse bursts (Fig.1).

An additional feature consisting of focusing MT pulses at the center of k-space, hence called partial Fourier (PF) MT excitation, was implemented to allow investigation of higher RF intensities and as a 
mean to reduce specific absorption rate (SAR) for high field applications (24). The phase-encoding view order was modified to elliptical centric-out, leading to Cartesian spiral trajectories such that lines of kspace were acquired by increasing Cartesian distance from the k-space center (see Appendix). Five seconds of dummy excitations (both MT and readout pulses) were played before the system started acquiring data from the k-space center, and the MT pulse intensity was set to zero after a given number of k-space lines were acquired (typically 50\%), such that the remaining outer k-space was sampled without playing further MT pulses. Preliminary validation of the PF saturation approach is presented in the Appendix.

\section{MR imaging experiments}

Four different experimental studies were performed with the proposed ihMT-GRE sequence: 1/ Study of the TR dependence of inMT at fixed time averaged power, i.e. of the boost-effect, for various number of pulses within each saturation burst, 2 / characterization of the power dependence of ihMT at different TR setting, i.e. for distributed and concentrated power deposition regimes, $\mathbf{3}$ / study of the offset-frequency dependency, and 4/ reproducibility of the optimal configuration identified from previous studies and performed in high resolution scans. All investigated saturation parameters are detailed in Table 1.

All acquisitions were performed in axial orientation ( $R-L$ phase encoding direction) covering the whole brain. Readout parameters for all studies (unless otherwise specified) were: $T E=4.8 \mathrm{ms,}$ $320 \times 200 \times 160 \mathrm{~mm}^{3}$ FOV, $128 \times 80 \times 64$ matrix (for a $2.5 \mathrm{~mm}$ isotropic resolution), $\mathrm{BW}=100 \mathrm{~Hz} / \mathrm{pixel}$, RF spoiling, partial Fourier readout (6/8 in both phase encoding directions), GRAPPA 2 in R-L (25). The readout flip angle (FA) values were set proportionally to the Ernst angle (see Appendix) and the number of readout segments was maximized whenever possible (Table 1). All MT weighted volumes were averaged twice (or thrice) for improved SNR, and a 3D volume was acquired with RF saturation power set to zero to enable calculation of quantitative ihMT ratios (ihMTR, Eq. 3).

For each volunteer, an additional $\mathrm{T}_{1}$-weighted MPRAGE dataset was acquired for anatomical reference and segmentation of brain structures $\left(T R / T E=1900 / 2.92 \mathrm{~ms}, T \mathrm{I}=1100 \mathrm{~ms}, \mathrm{BW}=130 \mathrm{~Hz} /\right.$ pixel and FA $=15^{\circ}$, 250mm FOV, 250x250 matrix, 1mm slices), allowing for automated analysis of ihMT data.

\section{Study 1: Optimization of the TR boost-effect for various numbers of ihMT pulses}

This study focused on optimizing the TR between RF saturation bursts composed of various numbers of pulses $\left(N_{P}=6,12\right.$ and 18$)$. The whole study was performed at constant SAR level (representing about 80- 
95\% of the SAR regulatory limitation, depending on the subject) and for two averaged RF intensities: $\mathrm{B}_{1 \mathrm{RMS}}=4.1 \mu \mathrm{T}$ (full $\mathrm{k}$-space saturation) and $\mathrm{B}_{1 \mathrm{RMS}}=5.5 \mu \mathrm{T}(50 \% \mathrm{PF}$ saturation). Therefore the peak RF amplitude $\left(B_{1}\right.$ Peak $)$ of individual saturation pulses was increased when TR was lengthened, ensuring constant power deposition over TR. The range of investigated TRs was dependent on $N_{P}$ and $B_{1 R M S}$, from the minimal achievable value imposed by timing constraints, to the maximal value imposed by hardware limitations (maximum $B_{1 \_ \text {Peak }}$ of $30 \mu \mathrm{T}$ for the body coil on the $1.5 \mathrm{~T}$ system).

\section{Study 2: Characterization of the power dependence of inMT}

The ihMT-GRE approach introduced mixing periods within the long MT saturation train. In addition, lengthening TR at constant SAR level required increased $B_{1 \_ \text {Peak }}$ of individual MT pulses and led to a more concentrated energy deposition scheme (EDS). In this study more insight into the mechanisms underlying the boost-effect was provided by studying inMT as a function of RF power, for different TR settings, i.e. for different EDS, and at fixed $N_{p}$. Three configurations were investigated: 1 / Shortest TR (24ms) corresponding to the most distributed EDS achievable with current sequence timing constraints, 2/ TR=67ms for an intermediate EDS, chosen from Study 1 to maximize ihMTR (ihMTR vs. TR at 5.5 $\mu$ T for $\left.N_{p}=12\right)$, and 3 / longest TR $(140 \mathrm{~ms})$ corresponding to the most concentrated EDS based on hardware limitations $\left(B_{1}{ }_{1}\right.$ Peak $)$. In the latter case it was necessary to use higher-power Tukey windowed pulses (see (26) with $\alpha=0.3$, normalized amplitude and power integrals of 0.85 and 0.81 , respectively) to allow exploration of the desired range of RF powers at such a long TR.

Results are expressed as a function of $\mathrm{B}_{1 \mathrm{RMS}}$ (defined over TR, and varied from $2 \mu \mathrm{T}$ to $5.5 \mu \mathrm{T}$ ), and as a

function of the saturation power defined over the ihMT pulse train $\left(\mathrm{B}_{1 \mathrm{SAT}}=\mathrm{B}_{1 \mathrm{RMS}} \sqrt{\mathrm{TR} /\left(\mathrm{N}_{\mathrm{P}} \Delta \mathrm{t}\right)}\right.$ ) (see Fig.1).

\section{Study 3: Influence of the frequency offset on ihMT signal}

Effects of the frequency-offset, $\Delta f$, were explored at two power levels, $B_{1 R M s}$ of $4.1 \mu \mathrm{T}$ and $5.5 \mu \mathrm{T}$ and for $N_{p}=6$ and 12. For each tested configuration TR was chosen from Study 1 to maximize ihMTR. $\Delta f$ was varied from $5 \mathrm{kHz}$ to $13 \mathrm{kHz}$ by $2 \mathrm{kHz}$ increment.

\section{Study 4: Robustness and reproducibility study}

In order to assess the reproducibility of the optimized ihMT-GRE protocol, the best configuration derived from previous studies (see Table 1) was repeated twice on three volunteers, with improved spatial

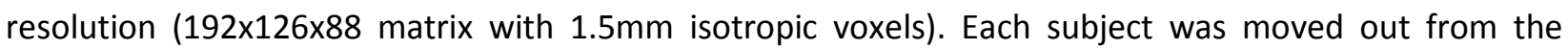


scanner after the first acquisition and repositioned on the bed a few minutes later for a second examination.

\section{Data processing}

MPRAGE data were first processed using FreeSurfer (27) for automatic segmentation of cortical gray matter (GM), white matter (WM) and Thalamus (Th) and for extraction of a whole brain mask (BM). Then MPRAGE volumes were registered to the MNI152 template space using the ANTs library (SyN registration (28)) and masks of Corpus Callosum (CC) and Pyramidal Tract (PT, cropped above internal capsule) were extracted from the JHU tract atlas (29). These masks were then warped back to the MPRAGE subject space. All volumes required to generate the composite ihMTR images were interpolated (zero-filling) and a cosine window function (26) was applied to remove ringing artefacts. The data were then motioncorrected using the realign SPM routine (30) prior to image combination. The ihMTR volume was subsequently co-registered to the MPRAGE reference frame using SPM in order to calculate histograms and statistics (mean +/- Standard Deviation) for the afore-mentioned masks (WM, GM, CC, PT, Th and BM) using Matlab (The MathWorks Inc., Natick, MA) custom routines. Finally, for each study, group statistics (average values and standard errors of the mean) are reported on the corresponding figures, calculated across volunteers for each brain area.

\section{Numerical simulations}

The presented ihMT theory (Eqs.1-3) allows calculation of the ihMT signal for various sequence variants providing that RF saturation, relaxation and signal readout phases are properly taken into account. Here, a piece-wise numerical integration was implemented using a Matlab solver (ode45), using analogous working hypothesis as done for conventional MT-GRE sequence by Sled and Pike (9). The MT shaped pulses were approximated by rectangular pulses of identical duration, frequency offset and average power. Both direct saturation and macromolecular saturation effects were considered during RF irradiation by accounting for the corresponding RF absorption terms. Conversely the readout flip angle was assumed to have negligible effect on the bound pool and instantaneous tipping of the free pool magnetization was assumed. In addition, following Varma et al. (11) the presented ihMT theory was further refined to account for two distinct bound pools: 1/ one including dipolar order and corresponding to a fraction $\mathrm{f}$ of the total bound protons, and $2 /$ the other which does not contain dipolar order and corresponding to the remaining fraction 1-f. Both bound pools were assumed to have identical 
$R_{1 B}, T_{2 B}$, lineshapes, and exchange rate $R$ with the free pool. In other words the dipolar relaxation $T_{1 D}$ is only associated with a fraction $f$ of the bound pool, making the two parameters $\left\{T_{1 D}, f\right\}$ the relevant variables to characterize the influence of dipolar order according to prior ihMT theory. Numerical integrations were run until a steady state was reached and the simulated ihMTR was calculated according to Eq.3 from the longitudinal magnetization obtained immediately before the readout pulse.

In the experimental studies, dual-offset frequency saturation was achieved by alternating the offset frequency of MT pulses. Strictly speaking, dual-frequency saturation was not performed simultaneously, and should not follow Eq. 2. Thus, the dipolar order is not decoupled from the Zeeman order during RF pulses for alternating dual-frequency experiments, and significant $T_{1 D}$-filtering may occur $(18,19)$, thereby reducing the intensity of the ihMT effect. For very short frequency switching times (i.e. $\Delta t \ll$ $T_{1 D}$ ) it may be assumed that dipolar order effects effectively vanish when averaged over one period of frequency alternation. Hence, for numerical simulations, Eq.1 was used with alternation of the offset frequency in order to mimic the dual-saturation applied in experiments.

Simulations considered the following tissue parameters, adapted from literature and based on fitting of human Internal Capsule (IC) data to simplified analytical ihMT models $(11,18): 1 / R_{1 A}=650 \mathrm{~ms}(31)$, $1 / R_{1 B}=1 s, R=65 s^{-1}, T_{1 D}=6.2 m s, f=0.65, T_{2 B}=12.5 \mu s, 1 /\left(R_{1 A} \cdot T_{2 A}\right)=581, R \cdot M_{O B} / R_{1 A}=7.3$. Studies 1 to 3 were simulated with sequence parameters as experimentally prescribed, except for the power dependence study (Study 2), for which slightly different TR values were simulated. These values were chosen based on simulation of ihMTR vs. TR at $5.5 \mu \mathrm{T}$ from Study 1: optimal TR of $90 \mathrm{~ms}$ for the intermediate EDS and TR of 180ms (i.e. 2 times longer, as for the experiments) for the concentrated EDS. Table 2 summarizes all simulated parameters.

\section{RESULTS}

For the sake of conciseness and figure readability, results and figures of the experimental studies mostly focus on PT which provides the highest inMT ratios; measurements performed in other brain areas are reported as supplementary material.

\section{TR boost-effect for various number of ihMT pulses}

Fig.2 shows the ihMTR results from changes in TR for various $N_{P}$ and $B_{1 R M S}$ values. At the lower energy level (4.1 $\mu$ T in Fig.2a), a broad range of TRs were investigated for $N_{p}=12$ and 18, revealing a 
characteristic shape that passes through a maximum. Similar trends could be extrapolated from the other curves, although not fully sampled due to $B_{1_{-} \text {Peak }}$ restrictions (Fig.2a $N_{P}=6$, and Fig.2c). At $4.1 \mu T$, $N_{P}$ of 6 and 12 yielded similar maximum ihMTR (around $13.5 \%$ in PT), although obtained for different TR settings. A lower maximum was obtained for $N_{P}=18$. At $5.5 \mu T, N_{P}=12$ yielded the maximum ihMTR around $15 \%$ in PT for TR = $67 \mathrm{~ms}$ and lower values were obtained for all other investigated configurations. Note that other brain areas demonstrated similar parameter dependence with the same characteristic shape, although with different absolute ihMTR values (see Fig.S1 and S2).

The corresponding simulations (Fig.2b,d) predicted similar observations with well depicted rising-andfalling curves and ihMTR close to that obtained experimentally. However the optimal TR values differed slightly, and the maximum ihMTR simulated with $\mathrm{N}_{P}=18$ at $4.1 \mu \mathrm{T}$ was very close to those simulated with $N_{p}=6$ or 12 (Fig.2b). Conversely the maximum ihMTR obtained with $N_{p}=18$ at $5.5 \mu$ T was slightly lower than for $N_{P}=12$, and simulations suggested that $N_{P}=6$ could perform slightly better than $N_{p}=12$ (Fig.2d) if the optimal TR and corresponding $B_{1 \_ \text {Peak }}$ could be reached experimentally.

\section{Power dependence of ihMT}

Fig. 3 focuses on the power dependence obtained for three different TR values, i.e. for three different energy deposition schemes. For the most distributed EDS (Fig.3a, orange line), the ihMTR increased slowly and almost linearly over the whole studied $B_{1 R M S}$ range. For the intermediate EDS (Fig.3a, brown line) the linear signal increase was faster up to $B_{1 R M S}=4 \mu \mathrm{T}$, then inflected towards a sub-linear dependency and tended to reach a maximum for the highest power level. Finally, for the concentrated EDS (Fig.3a, black line), the signal increase was rapid up to $B_{1 R M S}=3 \mu T$, followed by a strong inflexion leading to a plateau for $B_{1 R M S} \geq 4 u T$. Consistent with Study 1 (Fig.2), higher ihMTR is obtained when concentrating the EDS, especially at relatively low power, and the maximal inMTR was obtained for the intermediate EDS. Illustrative ihMTR images acquired for the three EDS at $\mathrm{B}_{1 \mathrm{RMS}}=5.5 \mu \mathrm{T}$ are displayed in Fig. S3. When expressed as a function of the saturation power $B_{15 A T}$ the results revealed similar patterns (Fig.3c) with a linear increase for relatively low powers followed by strong inflexion above $\mathrm{B}_{1 \mathrm{SAT}} \approx 10 \mu \mathrm{T}$, and eventually leading to saturation as observed for the concentrated EDS. Similar behaviors were obtained in all investigate brain areas albeit with different ihMTR intensities (see Fig.S4 and S5).

The simulations were in good agreement with experiments especially regarding the actual ihMTR values obtained over the investigated ranges for the distributed and intermediate EDS (Fig.3b and 3d). The same patterns are observed and further inferences could be made with a quasi-linear regime at relatively 
low $B_{1 R M S}$ followed by a curve inflexion (Fig.3b), with no actual saturation plateau shown from simulation. Instead the curves reached a maximum and decayed at higher power levels. The exploration of a wider range of RF power allowed by the simulations, especially when displayed as a function of $B_{1 S A T}$ (Fig.3d), further questioned the existence of such a saturation plateau, as well as common power dependence regimes for the different tested EDS since the curve inflexion appeared to occur for different $B_{1 S A T}$ values.

\section{Frequency offset dependency of ihMT}

The characteristic bell-shaped ihMTR dependence on the RF frequency-offset is shown in Fig.4. For all configurations tested, and in all brain areas, ihMTR was maximized when $\Delta f=7-9 \mathrm{kHz}$. For higher power the peak frequency at which the maximum occurred was shifted (within this range) to slightly larger offsets, as well as providing higher ihMTRs. Minor differences were observed for the two $N_{p}$ values explored, with TR adjusted from Study 1 to maximize ihMTR at $7 \mathrm{kHz}$. Simulations were in very good agreement. Other brain areas yielded similar frequency dependencies (see Fig.S6 and S7).; however the ihMTR and the curvature of the curves were somewhat different, especially for CC. Of interest, for all tested configurations PT and CC data crossed near $11 \mathrm{kHz}$.

\section{Optimized ihMT protocol for whole brain imaging at 1.5T}

Overall, studies 1-3 suggested that the following parameters should be used to maximize the ihMT sensitivity: $B_{1 R M S}=5.5 \mu \mathrm{T}$ using $50 \% P F, N_{P}=12, T R=67 \mathrm{~ms}$ and $\Delta f=8 \mathrm{kHz}$. Fig. 5 displays representative 3D inMTR images obtained with the optimized protocol and acquired with $1.5 \mathrm{~mm}$ isotropic resolution. On Fig.6a, the corresponding histograms of ihMTR values measured in selected brain areas are displayed showing an averaged ihMTR of up to $16 \%$ in PT. Finally the reproducibility is summarized on Fig.6b, showing very low variability of the mean ihMTR measured in all brain areas for all subject (intraindividual error $<2 \%)$. The corresponding inter-individual statistics indicated a relative variation of ihMTR lower than $10 \%$ among volunteers.

\section{DISCUSSION}

\section{Sequence optimization for whole brain imaging}


The proposed sensitivity-boosted and time-efficient steady-state ihMT-GRE sequence implemented and optimized at 1.5T, enabled high-resolution (1.5mm isotropic) 3D ihMT imaging of the whole brain within a clinically compatible scan time ( $15 \mathrm{~min})$. By taking advantage of mixing periods between saturation bursts it was possible to enhance the ihMT signal intensity by a factor of $\sim 2$ as compared to previously reported ihMT-prep implementations that use more distributed pulse train (2). The optimized approach yielded averaged ihMTRs in PT up to $16 \%$ and excellent reproducibility (Figs. 5 and 6). The average WMto-GM contrast estimated from the ratio of inMTRs measured in the whole WM and GM was 2.3, reaching 2.6 when considering the PT mask instead of the whole WM, in agreement with previous studies using ihMT-prep implementations (2). Of interest, the higher ihMTRs measured in WM at high resolution (compare Fig.5 vs Fig.2-4) may suggest partial volume effect contaminating low resolution data, and highlighted the need for highly resolved acquisitions. Of particular interest two features of the proposed sequence, namely partial k-space MT saturation and readout segmentation, allowed for more flexibility with SAR reduction and/or saturation power adjustment (see Appendix), ultimately allowing for higher ihMTR to be obtained, and for keeping scan time within reasonable limits. Finally, it is worth noting that GRE readout improvements, such has alternate parallel imaging sampling strategies, were not investigated in the current study and could further improve resolution and/or reduce scan time.

\section{ihMT dependence on saturation parameters}

Previous studies using ihMT-prep approaches with more distributed RF saturation pulses characterized the inMT signal dependence on saturation parameters $(1,2,11)$. The current findings obtained with the ihMT-GRE approach are in good agreement with those studies in terms of frequency offset and RF power. The optimal frequency offset for imaging CNS tissues was found to lie between 7-9 kHz for the investigated power range $\left(B_{1 R M S}=4-5.5 \mu \mathrm{T}\right.$, Fig.4), showing a slight increase with RF power but very little dependence on the number of pulses $\left(N_{P}\right)$ within a single RF burst. Noteworthy, a recent study (32) has demonstrated the sensitivity of ihMT with WM tract orientation with respect to the main magnetic field $B_{0}$. These effects, which are expected from the underpinning dipolar interaction, may be accounted for in the ihMT model and provide a reasonable description of the observed offset dependence of ihMT for WM tracts with distinct orientations, such at PT vs. CC. Concerning the RF power dependency of ihMT, although the EDS was found to be a determinant factor, , the quasi-linear relationship observed for the most distributed case (Fig.3) was consistent with measurements made using an ihMT-prep sequence with a highly distributed EDS (see Fig. $6 \mathrm{~b}$ in (2), the $1.3 \mathrm{~s}$ data approaches steady state conditions ; note that $\mathrm{B}_{1 \mathrm{RMS}}$ was defined in ref. (2) over a single ihMT pulse). 
This study did not investigate pulse width $(\mathrm{pw})$ and pulse repetition time $(\Delta \mathrm{t})$ effects, although they are of potential importance for ihMT optimization. A recent preclinical study has demonstrated the fundamental role of $\Delta t$ in driving the $T_{1 D}$ weighting of ihMT (19). Alternative ihMT implementations using a cosine modulation for the dual-frequency saturation provides different $T_{1 D}$ weighting cleared from any $\Delta t$ contribution. In practice this requires higher $B_{1}$ Peak and could suffer from SAR monitoring errors due to rapid RF modulation (33). Overall the settings used here constitute a good tradeoff providing high inMTR in CNS tissues with weak $T_{1 D}$ filtering (19), which may enhance specificity for myelinated tissues (34).

\section{A general ihMT simulation framework}

A general framework for ihMT modelling has been developed allowing for forward calculation of the inMT ratio obtained for different sequence variants. Numerical implementation was first validated by comparison with the analytical form of inMTR corresponding to the steady state following continuous wave RF excitation, as described in (11). Then, ihMT-GRE sequences with alternating frequency pulses for dual saturation were simulated for various experimental conditions and yielded consistent results in good agreement with experimental findings (Figs. 2-4). Most importantly the numerical simulations validated the boost-effect mechanism and related parameter dependencies in comparison with experiments. More generally, this framework can also be used to simulate cosine modulated pulses for simultaneous dual-frequency saturation by considering Eq.2 instead of Eq.1 for the dual saturation experiment.

To improve agreement with experiments, the model tissue parameters taken from IC data and the Morrison model (with variable f) in Varma et al (11) were adapted. In particular based on a recent study (18), which employed a modified ihMT sequence to estimate $T_{1 D}$ more accurately, a value of $6.2 \mathrm{~ms}$ was chosen for IC. Then, because the curvature of ihMTR vs. TR and the optimal TR value (Fig.2) appeared to be sensitive to $R$, its value was manually adjusted to $65 \mathrm{~s}^{-1}$. Other parameters listed in the method section were then determined by repeating the fitting process described in (11) using constrained $T_{1 D}, R$ and $f(f=0.65)$. Though the chosen parameters may not all reflect actual biophysical tissue properties (e.g. $R$ and $T_{2 a}$ usually demonstrate poor accuracy when estimated using the continuous wave approximation (35)) they provided satisfactory signal prediction in the context of the current study. Better agreement with experiment and a more accurate tissue characterization may be obtained using more comprehensive fitting approaches relying on all measured signal dependencies (ihMTR vs. $\Delta f, B_{1}, \Delta t$, TR...) and based on realistic ihMT models such as presented here. This warrants further studies. 


\section{Boosting the ihMT sensitivity using concentrated energy deposition}

An important mechanism driving signal optimization and leading to the TR boost-effect has been identified that relies on the dynamics of RF energy deposition. By properly tuning the TR and therefore the corresponding mixing time, the ihMTR can be maximized for given saturation parameters (Fig.2). Although at first glance such a sensitivity enhancement may be thought to originate from the stronger saturation power ( $\mathrm{B}_{1 \mathrm{SAT}}$ ) used at longer TRs, the power dependence study (Fig.3) teaches us that increasing RF power becomes counterproductive above a certain point, and that interleaving mixing periods with short saturation bursts allows a more efficient use of available RF energy. This effect was actually not initially expected since free relaxation periods were considered counterproductive for ihMT signal buildup because of the short lifetime of dipolar order, as in the case of long interpulse delays, i.e. long $\Delta t$ (19). Numerical simulations confirmed the optimal TR value's sensitivity, not only to $T_{1 D}$ but also to the exchange rate $R$, which taken together could explain the higher optimal TR values observed for simulations. Dependence on $\mathrm{R}$ indicates that the mixing periods incorporated into the ihMT saturation train are necessary to allow efficient propagation of the inMT effect all the way to the free pool, and may suggest that magnetization exchange is necessary to refresh the bound pool so the next RF burst can make efficient use of available RF energy. This interpretation is consistent with the power dependence study (Fig.3), which indicated that the optimal EDS was a tradeoff between RF power concentration and saturation of the ihMT effect. In addition, Fig.3 also suggests that the RF saturation power $\left(\mathrm{B}_{1 \mathrm{SAT}}\right)$ is highly relevant in ihMTR optimization, perhaps more so than average RF power. Overall, this study also shows that concentrated EDS provides enhanced ihMTR as compared to more distributed EDS, especially at relatively low power, a propitious finding in view of achieving inMT at higher fields. This sensitivity enhancement related to concentrated EDS would also apply to ihMT-prep sequences provided that mixing periods are incorporated within the long pre-saturation pulse train. This can be shown by numerical simulations and has been confirmed experimentally $(34,36-38)$.

Noteworthy, concentrating the RF energy and acquiring multiple k-space lines within a long TR may introduce undesired signal modulations in k-space. Indeed, within the proposed ihMT-GRE sequence the steady-state is dominated by the periodicity of the MT burst rather than the periodicity of the readout modules. In addition, the partial Fourier excitation and the phase-encoding view order further complexify the k-space modulation pattern which could potentially yield to ghosting artifacts or blurring. Although studying these effects and their relationship with the number of segments and nominal resolution is of particular interest, we simply report in this study that no obvious artifacts were observed 
in the final images. This may be explained by the combination of multiple intertwined mechanisms which taken altogether tend to mix and smooth out the effects of k-space modulation.

Finally, note that the dependence of the ihMTR on TR results from a combination of single- and dualfrequency MT saturation effects. This is illustrated in Fig.7, where simulations predict different dependencies at high RF saturation power whether cosine modulated pulses or frequency alternating pulses are used for dual-saturation. These could be explained considering that high saturation powers make the alternating dual saturation diverge from a genuine simultaneous dual saturation and progressively tend toward the single saturation scenario, hence reducing the ihMT effect. This is not surprising, since higher pulse power strengthens the coupling between Zeeman and dipolar orders (Eq. 1), eventually preventing efficient averaging of dipolar effects. These later considerations are of great importance when comparing different implementations of ihMT sequences.

\section{CONCLUDING REMARKS}

Optimizing ihMT acquisitions requires a comprehensive characterization of underlying signal dependence on saturation parameters. The present work has built upon previous characterization studies $(1,2,19)$ and has addressed the case of interleaved ihMT-GRE sequences for which mixing periods are incorporated into long ihMT saturation pulse train. A general simulation framework was presented and was shown to provide satisfactory predictions, which is very valuable for guiding sequence optimization. For experimental studies performed at 1.5T, optimal settings were presented and allow for high resolution whole brain acquisition in realistic scan time. Similarly, optimal settings accounting for specific limitations of higher field MRI systems, such as peak RF intensity and SAR, could be derived. For this purpose the influence of the energy deposition on the power dependence is of primary interest.

\section{APPENDIX}

\section{1/ Experimental validation of the partial Fourier saturation approach}

A first acquisition was performed with full $k$-space saturation and $B_{1 R M S}=4.1 \mu T$; then the acquisition was repeated using $50 \%$ PF saturation and identical $B_{1 R M S}$, hence leading to much reduced SAR (roughly halved), and using $50 \% \mathrm{PF}$ and increased $\mathrm{B}_{1 \mathrm{RMS}}$ of $5.7 \mu \mathrm{T}$, i.e. maintaining roughly constant SAR although 
using much stronger MT pulses. These acquisitions were performed on 3 volunteers (males, mean age = $30.1 \pm 10.3$ years) with $N_{p}=6$ Hann-shaped pulses, $\Delta f=7 \mathrm{kHz}, \mathrm{pw}=0.5 \mathrm{~ms}, \Delta \mathrm{t}=1 \mathrm{~ms}, \mathrm{TR}=19 \mathrm{~ms}$ ( 1 segment), and same readout parameters as for Study 1-3.

Assessment of the partial Fourier approach is presented in Fig.8. The 50\% PF saturation led to very similar ihMTR in all brain areas as compared to the full k-space saturation, with a maximum relative error of the mean of only $1.3 \%$ measured in whole WM, although the SAR was reduced by about $45 \%$ as compared to the full k-space saturation. When using 50\% PF and taking benefit of the SAR reduction to increase $B_{1 R M S}$, a significant enhancement of ihMTR was measured in all brain areas.

\section{2/Adjustment of the readout FA value with varying TRs and number of segments}

For adjustment of the readout FA at various TR, a preliminary study was conducted on one healthy volunteer (female, 34.6 y.o.) with the following inMT parameters: $N_{p}=12$ Hann-shaped pulses, $\Delta f=7 k H z$, $\mathrm{pw}=0.5 \mathrm{~ms}, \Delta \mathrm{t}=1 \mathrm{~ms}, \mathrm{TR}=50 \mathrm{~ms}$ ( 3 segments), $\mathrm{PF}=50 \%, \mathrm{~B}_{1 \mathrm{RMS}}=5.5 \mu \mathrm{T}$ and same readout parameters as for Study 1-3. FA was varied between $7^{\circ}$ and $15^{\circ}$ (for an estimated Ernst angle of $\sim 12.8^{\circ}$, accounting for the 3 segments, and assuming a $T_{1}$ of $650 \mathrm{~ms}$ (31)) and SNR was measured on the resulting ihMT image. The FA maximizing SNR of ihMT data was found to be $11^{\circ}$ (i.e. slightly lower - about $86 \%$ - than the Ernst angle), in agreement with previous findings related to conventional MT-GRE implementation (22). Note that although SNR was dependent on the readout FA ( 15\% relative SNR variation), the ihMTR showed very little variation (relative variation averaged over all brain areas $<2 \%$ ) with FAs over the investigated range. This is advantageous for the current characterization study as it further supports the use of the ihMTR metric to compare different saturation parameters, independently of SNR optimization. In all presented experiments, readout FAs were always adjusted to $~ 86 \%$ of the corresponding Ernst angle (assuming a $T_{1}$ of $650 \mathrm{~ms}$ ). For segmented acquisitions, FAs were adjusted to yield equivalent longitudinal magnetization attenuation effects during TR than would otherwise be obtained with a single readout.

\section{ACKNOWLEDGMENTS}

This study was supported by the ARSEP Foundation (Research Grant 2015), S.M received support from IRME 2016, V.H.P received support from A*MIDEX (grant $n^{\circ}$ ANR-11-IDEX-0001-02) funded by the French Government "Investissements d'Avenir" program. This work was performed by a laboratory member of France Life Imaging network (grant $n^{\circ}$ ANR-11-INBS-0006). Authors would like to thank Drs $B$. Robert and 
T. Troalen (Siemens Healthcare) for helpful discussions about sequence programming as well as $\mathrm{V}$. Gimenez, L. Pini, P. Viout and E. Soulier for volunteer management. 


\section{REFERENCES}

1. Varma G, Duhamel G, de Bazelaire C, Alsop DC. Magnetization Transfer from Inhomogeneously Broadened Lines: A Potential Marker for Myelin. Magn. Reson. Med. 2015;73:614-622. doi: 10.1002/mrm.25174.

2. Girard OM, Prevost VH, Varma G, Cozzone PJ, Alsop DC, Duhamel G. Magnetization transfer from inhomogeneously broadened lines (ihMT): Experimental optimization of saturation parameters for human brain imaging at 1.5 Tesla. Magn. Reson. Med. 2015;73:2111-2121. doi: 10.1002/mrm.25330.

3. Girard OM, Callot V, Prevost VH, Robert B, Taso M, Ribeiro G, Varma G, Rangwala N, Alsop DC, Duhamel G. Magnetization transfer from inhomogeneously broadened lines (ihMT): Improved imaging strategy for spinal cord applications. Magn. Reson. Med. 2017;77:581-591. doi: 10.1002/mrm.26134.

4. Korb J-P, Maruani J. Pulse saturation behavior of inhomogeneously broadened lines. I. Slow spectral diffusion case. Phys. Rev. B 1981;23:971-975. doi: 10.1103/PhysRevB.23.971.

5. Lee J-S, Khitrin AK, Regatte RR, Jerschow A. Uniform saturation of a strongly coupled spin system by two-frequency irradiation. J. Chem. Phys. [Internet] 2011;134. doi: 10.1063/1.3600758.

6. Morrison C, Stanisz G, Henkelman RM. Modeling magnetization transfer for biological-like systems using a semi-solid pool with a super-Lorentzian lineshape and dipolar reservoir. J. Magn. Reson. B 1995;108:103-113.

7. Sled JG, Pike GB. Quantitative Interpretation of Magnetization Transfer in Spoiled Gradient Echo MRI Sequences. J. Magn. Reson. 2000;145:24-36. doi: 10.1006/jmre.2000.2059.

8. Yeung HN, Adler RS, Swanson SD. Transient Decay of Longitudinal Magnetization in Heterogeneous Spin Systems under Selective Saturation. IV. Reformulation of the Spin-Bath-Model Equations by the Redfield-Provotorov Theory. J. Magn. Reson. A 1994;106:37-45. doi: 10.1006/jmra.1994.1004.

9. Sled JG, Pike GB. Quantitative imaging of magnetization transfer exchange and relaxation properties in vivo using MRI. Magn. Reson. Med. 2001;46:923-931.

10. Henkelman RM, Stanisz GJ, Graham SJ. Magnetization transfer in MRI: a review. NMR Biomed. 2001;14:57-64.

11. Varma G, Girard OM, Prevost VH, Grant AK, Duhamel G, Alsop DC. Interpretation of magnetization transfer from inhomogeneously broadened lines (ihMT) in tissues as a dipolar order effect within motion restricted molecules. J. Magn. Reson. 2015;260:67-76. doi: 10.1016/j.jmr.2015.08.024.

12. Swanson SD, Malyarenko DI, Fabiilli ML, Welsh RC, Nielsen J-F, Srinivasan A. Molecular, dynamic, and structural origin of inhomogeneous magnetization transfer in lipid membranes. Magn. Reson. Med. 2016:n/a-n/a. doi: 10.1002/mrm.26210.

13. Manning AP, Chang KL, MacKay AL, Michal CA. The physical mechanism of "inhomogeneous" magnetization transfer MRI. J. Magn. Reson. [Internet]. doi: 10.1016/j.jmr.2016.11.013. 
14. Chen J-H, Sambol EB, Decarolis P, O'Connor R, Geha RC, Wu YV, Singer S. High-resolution MAS NMR spectroscopy detection of the spin magnetization exchange by cross-relaxation and chemical exchange in intact cell lines and human tissue specimens. Magn. Reson. Med. 2006;55:1246-1256. doi: 10.1002/mrm.20889.

15. Davis JH, Auger M, Hodges RS. High resolution $1 \mathrm{H}$ nuclear magnetic resonance of a transmembrane peptide. Biophys. J. 1995;69:1917-1932. doi: 10.1016/S0006-3495(95)80062-7.

16. Taso M, Girard OM, Duhamel G, Le Troter A, Feiweier T, Guye M, Ranjeva J-P, Callot V. Tract-specific and age-related variations of the spinal cord microstructure: a multi-parametric MRI study using diffusion tensor imaging (DTI) and inhomogeneous magnetization transfer (ihMT). NMR Biomed. 2016;29:817-832. doi: 10.1002/nbm.3530.

17. Prevost VH, Girard OM, Varma G, Alsop DC, Duhamel G. Minimizing the effects of magnetization transfer asymmetry on inhomogeneous magnetization transfer (ihMT) at ultra-high magnetic field (11.75 T). Magn. Reson. Mater. Phys. Biol. Med. 2016:1-11. doi: 10.1007/s10334-015-0523-2.

18. Varma G, Girard OM, Prevost VH, Grant AK, Duhamel G, Alsop DC. In vivo measurement of a new source of contrast, the dipolar relaxation time, T1D, using a modified inhomogeneous magnetization transfer (ihMT) sequence. Magn. Reson. Med. 2016:n/a-n/a. doi: 10.1002/mrm.26523.

19. Prevost VH, Girard OM, Mchinda S, Varma G, Alsop DC, Duhamel G. Optimization of inhomogeneous magnetization transfer (ihMT) MRI contrast for preclinical studies using dipolar relaxation time (T1D) filtering. NMR Biomed. [Internet] 2017. doi: 10.1002/nbm.3706.

20. Varma G, Schlaug G, Alsop DC. 3D Acquisition of the Inhomogeneous Magnetization Transfer Effect for Greater White Matter Contrast. In: Proceedings of the ISMRM. Salt Lake City, Utah, USA; 2013. p. 4224.

21. Girard OM, Le Troter A, Varma G, Prevost VH, Guye M, Ranjeva J-P, Alsop DC, Duhamel G. Whole Brain inhomogeneous MT using an ihMT prepared 3D GRE sequence at 1.5T. In: Proceedings of the ISMRM. Toronto, Canada; 2015. p. 3356.

22. Pike GB. Pulsed magnetization transfer contrast in gradient echo imaging: A two-pool analytic description of signal response. Magn. Reson. Med. 1996;36:95-103.

23. Girard OM, Varma G, Mchinda S, Prevost VH, Le Troter A, Rapacchi S, Guye M, Ranjeva J-P, Alsop DC, Duhamel G. Theoretical and Experimental Optimization of a 3D Steady-State Inhomogeneous Magnetization Transfer (ihMT) Gradient Echo Sequence: Boosting the ihMT Sensitivity with Sparse Energy Deposition. In: Proceedings of the ISMRM. Singapore; 2016. p. 2892.

24. Lin C, Bernstein MA, Gibbs GF, Huston J. Reduction of RF power for magnetization transfer with optimized application of RF pulses in k-space. Magn. Reson. Med. 2003;50:114-121. doi: 10.1002/mrm.10513.

25. Griswold MA, Jakob PM, Heidemann RM, Nittka M, Jellus V, Wang J, Kiefer B, Haase A. Generalized autocalibrating partially parallel acquisitions (GRAPPA). Magn. Reson. Med. 2002;47:1202-1210. doi: 10.1002/mrm.10171. 
26. Harris FJ. On the use of windows for harmonic analysis with the discrete Fourier transform. Proc. IEEE 1978;66:51-83.

27. https://surfer.nmr.mgh.harvard.edu/. FreeSurfer.

28. Avants BB, Epstein CL, Grossman M, Gee JC. Symmetric diffeomorphic image registration with crosscorrelation: evaluating automated labeling of elderly and neurodegenerative brain. Med. Image Anal. 2008;12:26-41. doi: 10.1016/j.media.2007.06.004.

29. Hua K, Zhang J, Wakana S, Jiang H, Li X, Reich DS, Calabresi PA, Pekar JJ, van Zijl PCM, Mori S. Tract probability maps in stereotaxic spaces: analyses of white matter anatomy and tract-specific quantification. Neurolmage 2008;39:336-347. doi: 10.1016/j.neuroimage.2007.07.053.

30. http://www.fil.ion.ucl.ac.uk/spm/. Statistical Parametric Mapping.

31. Rooney WD, Johnson G, Li X, Cohen ER, Kim S-G, Ugurbil K, Springer CS. Magnetic field and tissue dependencies of human brain longitudinal $1 \mathrm{H} 2 \mathrm{O}$ relaxation in vivo. Magn. Reson. Med. 2007;57:308318. doi: $10.1002 / \mathrm{mrm} .21122$.

32. Girard OM, Prevost VH, Mchinda S, Varma G, Alsop DC, Duhamel G. Anisotropy of inhomogeneous Magnetization Transfer (ihMT) in White Matter. In: Proceedings of the ISMRM. Honolulu, HI, USA; 2017. p. 472.

33. Vu AT, Auerbach E, Lenglet C, Moeller S, Sotiropoulos SN, Jbabdi S, Andersson J, Yacoub E, Ugurbil K. High resolution whole brain diffusion imaging at 7T for the Human Connectome Project. Neurolmage 2015;122:318-331. doi: 10.1016/j.neuroimage.2015.08.004.

34. Prevost VH, Girard OM, Cayre M, Varma G, Mchinda S, Ranjeva J-P, Pelletier J, Durbec P, Alsop DC, Duhamel G. Validation of inhomogeneous Magnetization Transfer (ihMT) as a myelin biomarker. In: Proceedings of the ISMRM. Honolulu, HI, USA; 2017.

35. Portnoy S, Stanisz GJ. Modeling pulsed magnetization transfer. Magn. Reson. Med. 2007;58:144-155. doi: $10.1002 / \mathrm{mrm} .21244$.

36. Varma G, Girard OM, Prevost VH, Duhamel G, Alsop DC. Increasing the inhomogeneous magnetization transfer (ihMT) signal in vivo with high amplitude, low duty cycle irradiation. In: Proceedings of the ISMRM. Singapore; 2016. p. 2890.

37. Varma G, Grant AK, Girard OM, Prevost VH, Duhamel G, Alsop DC. Modeling the increased inhomogeneous magnetization transfer (ihMT) signal from high amplitude, low duty cycle irradiation. In: Submitted to ISMRM. Honolulu, HI, USA; 2017.

38. Prevost VH, Girard OM, Mchinda S, Varma G, Alsop DC, Duhamel G. On the boost effect of inhomogeneous Magnetization Transfer (ihMT). In: Proceedings of the ISMRM. Honolulu, HI, USA; 2017. 


\section{FIGURE CAPTIONS}

Figure 1: ihMT-GRE sequence kernel diagram. A pulsed ihMT module, consisting of a burst of $N_{p}$ consecutive off-resonance pulses, is interleaved with a conventional GRE readout. In the current study dual-frequency saturation is achieved by alternating the frequency-offset $( \pm \Delta f)$ of consecutive MT pulses. $T_{S A T}$ is the duration of the ihMT module, and $B_{1 S A T}$ the root mean square RF intensity delivered over this period of time. TR is the repetition time of the whole sequence kernel, with $B_{1 R M S}$ the corresponding root mean square RF intensity. Multiple lines of k-space (readout segments) may be acquired consecutively within a single kernel repetition in order to maintain scan time within clinically acceptable limits for long TRs.

Figure 2: Optimization of the TR boost-effect for various numbers of ihMT pulses, $N_{p}$, and two $B_{1 R M S}$ values. Panels (a) and (c) display experimental values of ihMTR vs. TR acquired at constant average RF power, and measured in the pyramidal tract (PT). As TR increases the peak RF intensity of MT pulses is increased in order to maintain $B_{1 R M S}$. The range of investigated TR values was adjusted for each parameter set $\left(N_{P}\right.$ and $\left.B_{1 R M S}\right)$, from the minimal achievable value, imposed by the sequence timing constraints, to the maximal value, imposed by hardware limitations (maximum peak RF intensity of $\approx 30 \mu \mathrm{T}$ ). Reported ihMTR values and errors bars correspond to the mean and group standard deviation for $n=3$. Corresponding numerical simulations (b) and (d), following the Provotorov theory of RF saturation and performed using methods and tissue parameters described in the text. Detailed parameters are provided in Tables 1 and 2.

Figure 3: Characterization of the power dependency regimes of ihMT for various Energy Deposition Scheme (EDS). The distributed and concentrated EDS were imposed by sequence timing and hardware (peak RF intensity) limitations, whereas the intermediate EDS was selected from Study 1 (Fig.2) as the configuration that maximizes ihMTR. Experimental values measured in the pyramidal tract (PT) and corresponding simulations are displayed as a function of $B_{1 R M S}$ in (a) and (b), respectively, and as function

of $B_{1 S A T}$ in (c) and (d), respectively, with $B_{1 S A T}=B_{1 R M S} \sqrt{T R /\left(N_{P} \Delta t\right)}$. Illustrative ihMTR images acquired for the three EDS at $B_{1 R M S}=5.5 \mu \mathrm{T}$ are reported in Fig. S3. For experimental data the reported ihMTR values and errors bars correspond to the mean and group standard deviation for $n=3$. Detailed parameters are provided in Tables 1 and 2.

Figure 4: Influence of the frequency-offset on ihMT signal. Experimental values measured in the pyramidal tract (PT), (a) and (c), and corresponding simulations, (b) and (d), are displayed vs. the off- 
resonance frequency $\Delta f$, for various numbers of ihMT pulses, $N_{P}$, and $B_{1 R M s}$ values. For experimental data the reported inMTR values and errors bars correspond to the mean and group standard deviation for $\mathrm{n}=3$. Detailed parameters are provided in Tables 1 and 2.

Figure 5: Demonstration of the optimized ihMT whole brain acquisition. Illustrative slices from a whole brain dataset showing very good contrast for myelinated tissues. The corresponding quantitative analysis is reported in Fig. 6. Conversely to previous optimization studies (Figs.2-4), the current protocol was run with a high spatial resolution $(1.5 \mathrm{~mm}$ isotropic vs. $2.5 \mathrm{~mm}$ isotropic previously). Detailed acquisition parameters are provided in Table 1. The Fig. 6

Figure 6: Quantitative analysis and reproducibility of the optimized ihMT whole brain acquisition. Sample histograms corresponding to the images from Fig. 5 are displayed in (a). Test-retest quantitative assessment of the protocol displayed in (b) was performed on 3 healthy volunteers, showing very good reproducibility, and relatively low inter-subject variation.

Figure 7: Insight into the fundamental difference between cosine-modulated and frequency alternating ihMT variants used for the dual-frequency saturation, according to simulations. The frequency alternating data shown on panels (a) and (b) are identical to those from Fig.3 (see corresponding captions). Here similar ihMT simulations using the cosine-modulated approach (see text) are displayed for comparison. Of interest, frequency alternating data appear to deviate further from cosine-modulated data at high saturation power (high $\mathrm{B}_{1 \mathrm{SAT}}$ values in (b)).

Figure 8: Assessment of the partial Fourier (PF) saturation approach. (a) Schematic representation of the elliptical centric-out Cartesian spiral trajectory used for the ihMT-GRE sequence readout in order to enable PF saturation. The phase and partition k-space grid (materialized by black dots) is sampled from the center of k-space by increasing Cartesian distance (materialized here by concentric circles). Following dummy scans, the center of $k$-space is acquired with ihMT pulses on (red area), until a given percentage of the whole k-space grid is acquired; and then, the peripheral k-space is acquired with the amplitude of ihMT pulses set to zero (blue area). (b) Quantitative assessment of the PF approach. The reported ihMTR values and errors bars correspond to the mean and group standard deviation for $n=3$.

\section{SUPPLEMENTARY FIGURE CAPTIONS}


Figure S1: Optimization of the TR boost-effect at $B_{1 R M S}=4.1 \mu T$, for various numbers of ihMT pulses, $N_{p}$. Data corresponding to the whole white matter mask (WM), to cortical gray matter (GM), to thalamus (Th) and to corpus callosum (CC) are displayed on panels a), b), c) and d), respectively. The reported inMTR values and errors bars correspond to the mean and group standard deviation for $n=3$. Data measured in the pyramidal tract (PT) are shown in Fig.2.

Figure S2: Optimization of the TR boost-effect at $B_{1 R M S}=5.5 \mu \mathrm{T}$, for various numbers of ihMT pulses, $N_{p}$. Data corresponding to the whole white matter mask (WM), to cortical gray matter (GM), to thalamus (Th) and to corpus callosum (CC) are displayed on panels a), b), c) and d), respectively. The reported inMTR values and errors bars correspond to the mean and group standard deviation for $n=3$. Data measured in the pyramidal tract (PT) are shown in Fig.2.

Figure S3: Illustrative inMTR images acquired with three different energy deposition schemes (EDS) and at $\mathrm{B}_{1 \mathrm{RMS}}=5.5 \mu \mathrm{T}$. The three EDS correspond to the ones from Study 2 and characterized in Fig 3.

Figure S4: Characterization of the power dependency regimes of ihMT for various Energy Deposition Scheme (EDS). Results are expressed in terms of averaged power, $B_{1 R M S}$, with data corresponding to the whole white matter mask (WM), cortical gray matter (GM), thalamus (Th) and corpus callosum (CC) displayed on panels a), b), c) and d), respectively. The three EDS correspond to the ones from Study 2 with detailed parameters provided in Tables 1 . The reported ihMTR values and errors bars correspond to the mean and group standard deviation for $n=3$. Data measured in the pyramidal tract (PT) are shown in Fig.3.

Figure S5: Characterization of the power dependency regimes of ihMT for various Energy Deposition Scheme (EDS). Results are expressed in terms of saturation power, $\mathrm{B}_{1 \mathrm{SAT}}$, with data corresponding to the whole white matter mask (WM), cortical gray matter (GM), thalamus (Th) and corpus callosum (CC) displayed on panels a), b), c) and d), respectively. The three EDS correspond to the ones from Study 2 with detailed parameters provided in Tables 1 . The reported ihMTR values and errors bars correspond to the mean and group standard deviation for $n=3$. Data measured in the pyramidal tract (PT) are shown in Fig.3.

Figure S6: Influence of the frequency-offset on ihMT signal for $N_{p}=12$ and two $B_{1 R M S}$ values. Data corresponding to the whole white matter mask (WM), cortical gray matter (GM), thalamus (Th) and corpus callosum (CC) are displayed on panels a), b), c) and d), respectively. The reported ihMTR values 
and errors bars correspond to the mean and group standard deviation for $n=3$. Data measured in the pyramidal tract (PT) are shown in Fig.4.

Figure S7: Influence of the frequency-offset on ihMT signal for $B_{1 R M S}=4.1 \mu T$ and two $N_{p}$ values. Data corresponding to the whole white matter mask (WM), cortical gray matter (GM), thalamus (Th) and corpus callosum (CC) are displayed on panels a), b), c) and d), respectively. The reported ihMTR values and errors bars correspond to the mean and group standard deviation for $n=3$. Data measured in the pyramidal tract (PT) are shown in Fig.4. 